\title{
Where in the Brain do Strokes Occur? A Pilot Study and Call for Data
}

Larry Nichols, MD; Joshua C. Bridgewater, BS; Nicholas B. Wagner, BS; Mathew Karivelil, BS; Himara Koelmeyer, BS; Denise Goings, MSN; and Thomas D. Hope, MD

Background: Every year, more than 795,000 people in the United States have a stroke, and each year about 140,000 Americans die from strokes. Although there is abundant information on the epidemiology, risk factors, pathophysiology, and many clinical features of strokes, there is a lack of specific numerical quantitation on the neuroanatomical distribution of strokes.

Methods: This retrospective study utilized de-identified reports of radiologic imaging to determine the neurovascular anatomical location of acute ischemic cerebral infarcts in a 637-bed regional hospital in a rural area during the one-year interval from October I, 2018 through September 30, 2019.

Results: During the one-year study period, there were 418 acute ischemic strokes, and $54.6 \%$ (228/4/8) were in the territory of a single large vessel. Of the single large vessel strokes, $62.3 \%$ (I42/228) were in a middle cerebral artery (MCA) territory, $12.1 \%(29 / 228)$ in a posterior cerebral artery (PCA) territory, 8.8\% (20/228) in a basilar artery distribution, $7.5 \%(17 / 228)$ in a posterior inferior cerebellar artery (PICA) distribution, 6.6\% (I5/228) in an anterior cerebral artery (ACA) distribution, I.8\% (4/228) in a superior cerebellar artery (SCA) distribution, and $0.4 \%(1 / 228)$ in an anterior inferior cerebellar artery (AICA) territory. Internal capsule lacunar strokes accounted for $17.7 \%$ (74/4I8) of the total, brainstem lacunar strokes for 8.1\% (34/4I8) and thalamic lacunar strokes for $5 \%(2 I / 4 I 8)$ of the infarctions. Watershed infarctions accounted for $2.9 \%(12 / 418)$ of the strokes, 9 in the MCA/PCA watershed area and 3 in the MCA/ACA watershed area.

Conclusions: This pilot study illustrates a methodology for collecting data to substitute specific numerical quantitation for vague generalities about the neuroanatomical distribution of strokes. Such quantification can enable evidence-based data-driven improvements in the care of stroke patients.

Keywords: Stroke; Cerebral Infarction; Neuroradiology, Data Collection

Corresponding Author: Larry Nichols, MD, Department of Pathology, Mercer University School of Medicine, I50I Mercer University Drive, Macon, GA 31207, Tel: (478) 30I-2405, Email: nichols_|@mercer.edu
Received: October 8, 2020

Revised: January |8, 202|

Accepted: March 26, 202।

doi: $10.3121 / \mathrm{cmr} .2021 .1632$ 
$\mathrm{S}$ trokes are common and can be fatal. Every year, more than 795,000 people in the United States have a stroke, and each year about 140,000 Americans die from strokes. Someone in the United States has a stroke every 40 seconds, and someone dies of stroke every 4 minutes. ${ }^{1}$ The etiology of strokes is evolving, and COVID-19 has emerged as a cause of stroke. ${ }^{2}$ Thromboemboli from the heart, often related to atrial fibrillation, are an increasing cause of stroke. ${ }^{3}$ Pregnancy is increasingly complicated by stroke. ${ }^{4}$

The etiology of a stroke correlates with its neuroanatomical location in the brain, clinical presentation, and appropriate treatment. $^{5}$ Appropriate treatment of stroke is a matter of extreme urgency because neurons die rapidly, within minutes of losing their blood supply. New treatments such as endovascular thrombectomy have significantly improved the outcomes of some types of stroke. ${ }^{5}$ Research into new stroke etiologies and treatments would benefit from precise data on the neuroanatomical location of strokes in the brain. Textbooks and review articles about stroke have abundant information on the epidemiology, risk factors, pathophysiology, and many clinical features, but lack specific numerical quantitation on the neuroanatomical distribution of strokes. This pilot study was undertaken to get a start on collecting the data needed and provide a model for how to do it.

\section{Methods}

This retrospective study was performed using de-identified neuroradiological imaging from the stroke center database of a 637-bed regional hospital within what is considered the stroke belt of the Southeastern United States. This database included all patients who were evaluated for an acute stroke during a one-year time period from October 1, 2018 through September 30, 2019. The study was limited to acute ischemic strokes and excluded hemorrhagic strokes. Imaging studies used included head computed tomography (CT), head and neck computed tomographic angiography (CTA), head magnetic resonance imaging (MRI), and head and neck magnetic resonance angiography (MRA). Head MRI was the preferred imaging study to determine the site of the acute stroke, but in instances in which an MRI was not performed, the other imaging modalities, generally head CT with or without head and neck CTA, were used to classify the stroke. The imaging studies were all interpreted by a radiologist while the patient was in the hospital. A board-certified neurologist then reviewed the images during our study to confirm the vasculature involved. Additionally, the neurologist confirmed if the imaging abnormalities present on scans matched the neurological presentation prior to the scan.

The strokes were classified by neuroanatomical vascular territory into the following categories: single large vessel, multiple large vessels, multiple small emboli, single large vessel and multiple small emboli, internal capsule lacunar, thalamic lacunar, brainstem lacunar, watershed, watershed with multiple small emboli, and watershed and single large vessel following textbook categorization of clinical syndromes correlated with pathology and imaging. ${ }^{6-8}$ The vessels classified as large were anterior cerebral artery (ACA), middle cerebral artery (MCA), posterior cerebral artery (PCA), superior cerebellar artery (SCA), anterior inferior cerebellar artery (AICA), posterior inferior cerebellar artery (PICA), and basilar artery.

If the imaging showed evidence of stroke in a vascular territory supplied by one large artery (left or right MCA, left or right PCA, etc.), it was labeled as a single large vessel stroke. If imaging revealed multiple small emboli within the tissue supplied by a single large vessel, then it was also considered to be a single large vessel stroke. Strokes that involved territory of two distinct large vessels, such as a simultaneous infarction in right PICA and left MCA territories, were classified as multiple large vessel strokes. The multiple small emboli classification was used if imaging revealed emboli in more than one vascular distribution without clear signs or symptoms of infarction in the territory of a single large vessel. A stroke was assigned to the large vessel and multiple small emboli category when imaging revealed an infarction of a territory supplied by a large artery, which was causing a deficit, with simultaneous emboli present in an area not supplied by the symptomatic artery. An example of a large vessel and multiple small emboli would be radiographic evidence of a right PCA stroke in a patient with a left visual field deficit, also with small and likely subclinical emboli scattered within the distribution of right and left MCA.

The internal capsule lacunar category was used when imaging showed evidence of a small stroke within the internal capsule. The thalamic lacunar category was used when imaging showed evidence of a small stroke within the thalamus. The brainstem lacunar classification was used when imaging revealed a single, small infarct in the territory of a penetrating artery of the brainstem. A stroke was assigned to the internal capsule lacunar and multiple small emboli category when imaging revealed an infarction of the internal capsule with emboli scattered within a different vascular territory or territories. The thalamic lacunar and multiple small emboli category was used when imaging revealed infarction of the thalamus with multiple small emboli in a different vascular territory or territories. If imaging showed evidence of infarction in an area partially supplied by two contiguous major vessels, it was assigned to the watershed stroke category. The watershed infarction with multiple small emboli category was used for infarctions of a watershed area with simultaneous emboli present in a separate vascular distribution, not in the watershed area. The watershed infarction and single large vessel classification was used when an infarct involved a watershed area and simultaneous infarction of a single large vessel territory not in the watershed area. Each individual stroke was also classified as on either the left or the right side of the brain. 

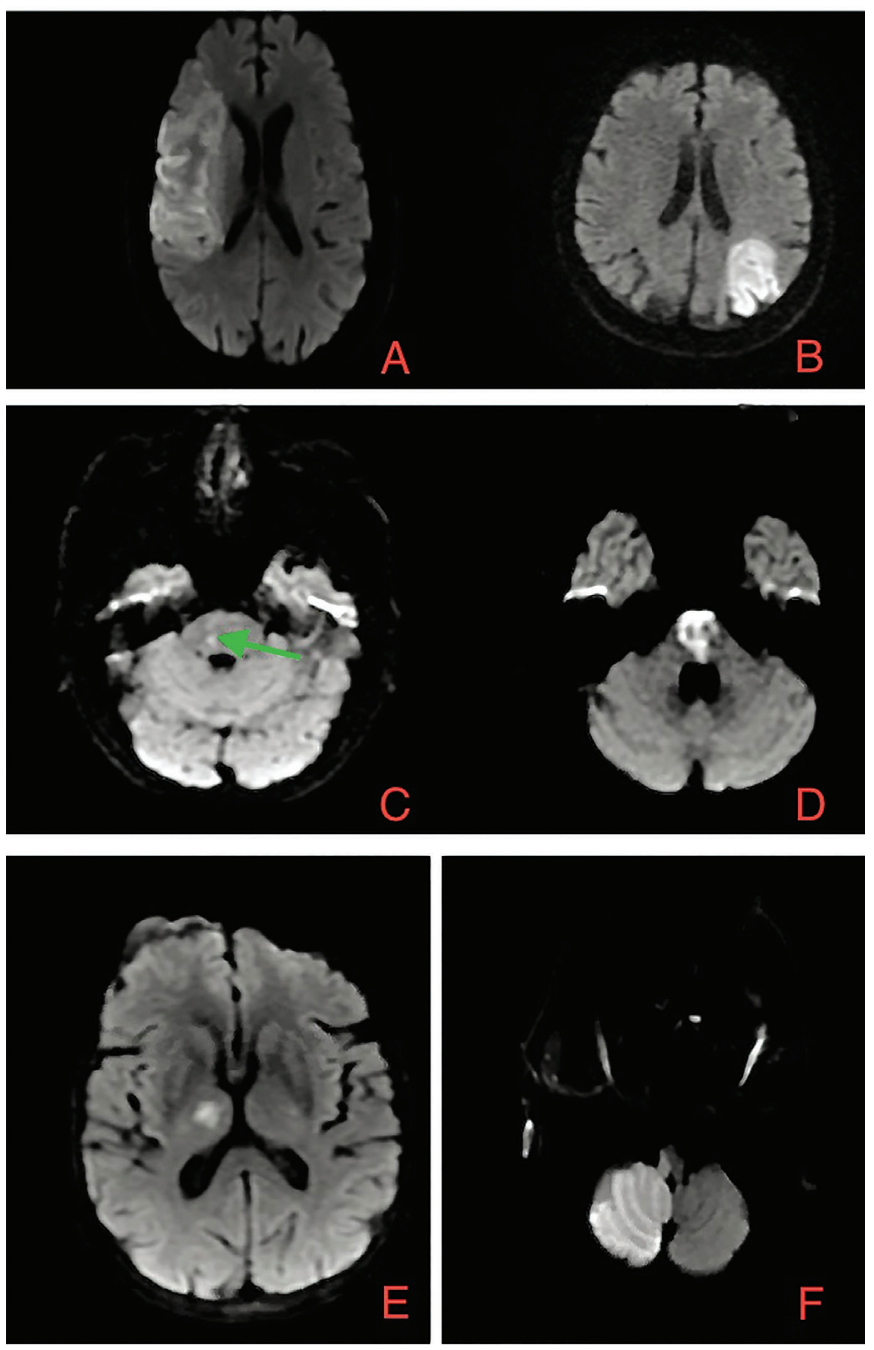

Figure 1. Magnetic resonance imaging (MRI) of acute ischemic strokes in right middle cerebral artery (MCA) territory (A), watershed between left MCA and posterior cerebral artery $(\mathrm{PCA})$ distributions $(\mathrm{B})$, right brainstem $(\mathrm{C})$, basilar artery territory $(D)$, right thalamus $(E)$, and posterior inferior cerebellar artery (PICA) territory $(\mathrm{F})$.
Infarctions that included more than one vascular distribution were tallied as a bilateral infarct only if the infarcts occurred in separate hemispheres of the brain. Infarction involving a single vascular distribution and multiple small emboli were considered a bilateral infarct if the emboli occurred in the opposite hemisphere as the large single vessel infarct. Basilar artery vascular distribution infarcts were placed in the bilateral category by default. Infarcts occurring in the SCA and AICA cerebellar circulations were lumped together in the other cerebellar category. This study was approved by the Institutional Review Board of the hospital.

\section{Results}

During the study period, 527 patients were admitted with a diagnosis of stroke. Of the 527 patients, however, 107 (20.7\%) had "no acute stroke" by imaging, and two cases had no imaging available to review, which left a total of 418 strokes that were categorized by neuroanatomical vascular territory. Examples of the neuroanatomical vascular territory localization are illustrated in Figure 1.

Of the 418 strokes, $228(54.6 \%)$ were in the territory of a single large vessel. Of the single large vessel strokes, 142 $(62.3 \%)$ were in an MCA territory, $29(12.1 \%)$ in a PCA territory, $20(8.8 \%)$ in a basilar distribution, $17(7.5 \%)$ in a PICA distribution, 15 (6.6\%) in an ACA distribution, 4 (1.8\%) in an SCA territory, and $1(0.4 \%)$ in an AICA territory. Brainstem lacunar strokes accounted for 34 (8.1\%) of the 418 total, internal capsule lacunar strokes for $74(17.7 \%)$, and thalamic lacunar strokes for $21(5 \%)$ of the infarctions. Watershed infarctions accounted for $12(2.9 \%)$ of the strokes - 9 in the MCA/PCA watershed area and 3 in the MCA/ACA watershed area. Of the 418 infarctions, 9 (2.2\%) were multiple large vessel strokes and multiple small emboli accounted for $29(6.9 \%)$ of the 418 strokes studied. The proportions of the strokes in these various categories are represented visually in Figure 2.

\section{Stroke Categories}

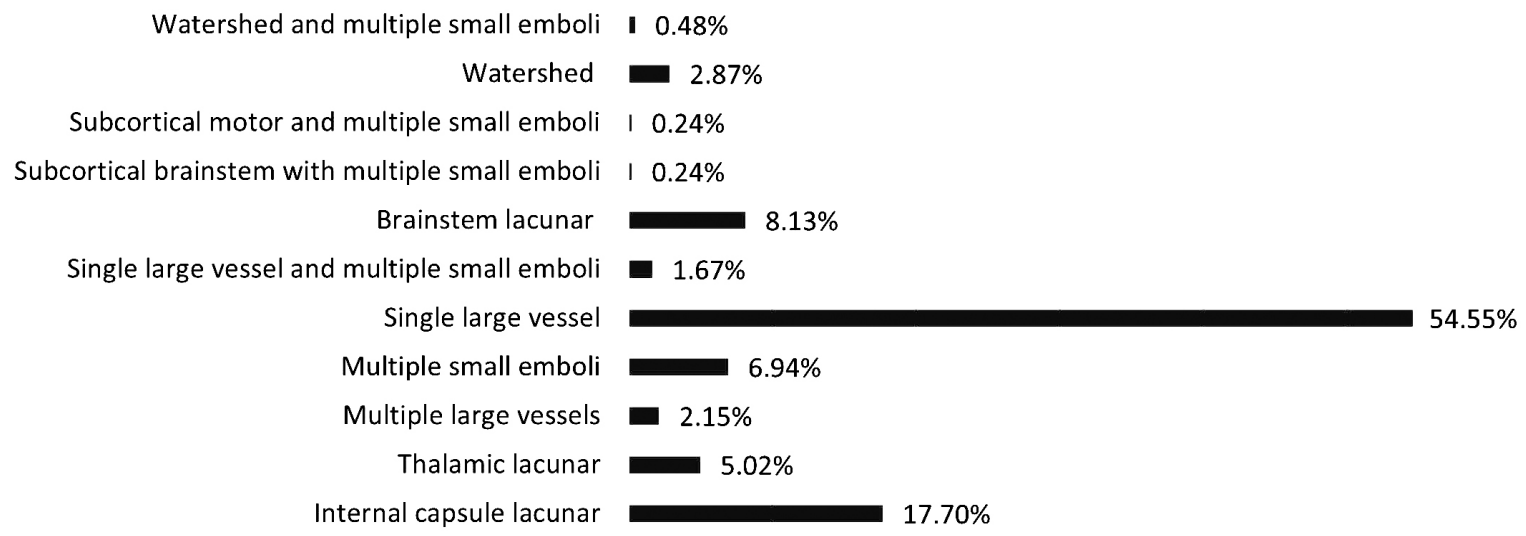

Figure 2. Stroke Categories 
Table 1: Anterior circulation infarcts. Infarctions involving the ACA (anterior cerebral artery), MCA (middle cerebral artery) or internal capsule region. See text for further description of categories.

\begin{tabular}{lccccc}
\hline Category & Left anterior & $\begin{array}{c}\text { Right } \\
\text { anterior }\end{array}$ & $\begin{array}{c}\text { Bilateral } \\
\text { anterior }\end{array}$ & $\begin{array}{c}\text { Total } \\
\text { anterior }\end{array}$ & $\begin{array}{c}\text { Percent of all 418 } \\
\text { infarcts }\end{array}$ \\
\hline ACA & 10 & 5 & 0 & 15 & $3.59 \%$ \\
ACA and MCA & 3 & 1 & 0 & 4 & $0.96 \%$ \\
ACA/MCA watershed & 1 & 2 & 0 & 3 & $0.72 \%$ \\
Bilateral ACA and left MCA & 0 & 0 & 1 & $0.24 \%$ \\
MCA & 68 & 74 & 0 & 142 & $33.97 \%$ \\
MCA with multiple small emboli & 0 & 1 & 4 & 5 & $1.20 \%$ \\
Internal capsule lacunar & 39 & 35 & 0 & 74 & $17.70 \%$ \\
Internal capsule lacunar with multiple & 1 & 0 & 0 & 1 & $0.24 \%$ \\
small emboli & & & &
\end{tabular}

ACA, anterior cerebral artery; MCA, middle cerebral artery

Single large vessel ischemic strokes approximated equal distribution between left and right sides of the brain. The laterality of the anterior circulation infarcts is shown in Table 1, the laterality of the posterior circulation infarcts in Table 2, and the laterality of those involving both anterior and posterior circulations in Table 3. Right MCA infarctions accounted for 74 (52.1\%), left $68(47.9 \%)$ of the 142 MCA strokes. Right PCA infarctions accounted for 13 (44.8\%), left 15 (51.7\%), and bilateral $1(3.4 \%)$ of the 29 PCA strokes. Right ACA strokes accounted for $5(33.3 \%)$ and left $10(66.7 \%)$ of the small total of 15 ACA infarctions. Strokes within the territories of multiple large vessels involved the following combinations: PCA and MCA (n=3); ACA and MCA $(n=4)$; PICA and MCA $(n=1)$. The category of single large vessel and multiple small emboli stroke accounted for $8(1.9 \%)$ of the 418 infarctions. Of these strokes, MCA with multiple small emboli accounted for $62.5 \%(n=5 / 8)$, PCA with multiple small emboli accounted for $25 \%(n=2 / 8)$, and PICA with multiple small emboli accounted for $12.5 \%(n=1 / 8)$ of the infarctions. There were two strokes $(0.5 \%)$ in the category of internal capsule lacunar infarct and multiple small emboli. Further details of the neuroanatomical vascular distribution of the strokes are displayed in Tables 1, 2, and 3.

In basilar artery infarcts, MRI almost always demonstrated multiple bilateral areas of acute infarction throughout the brainstem, often with some areas of cerebellar infarction; if done, CTA/MRA evidence of severe basilar disease was also present. Many PICA infarcts had CTA/MRA evidence of ipsilateral vertebral artery disease, which were categorized as a PICA infarct, not a vertebral artery infarct. Likewise, many MCA infarcts had CTA/MRA evidence of severe ipsilateral carotid disease, which were classified as MCA infarctions.

\section{Discussion}

Quantitative data are superior to descriptive adjectives like "common" or "rare". For stroke specifically, quantitative data from longitudinal population-based incidence studies with full case-ascertainment and repeated outcome assessments over time are the gold standard for outcome assessment. ${ }^{9}$ For

Table 2: Posterior circulation infarcts. Infarctions involving the basilar artery, PCA (posterior cerebral artery), PICA (posterior inferior cerebellar artery), other vasculature of the cerebellum, brainstem region or thalamic region. See text for further description of categories.

\begin{tabular}{lccccc}
\hline Category & $\begin{array}{c}\text { Left } \\
\text { posterior }\end{array}$ & $\begin{array}{c}\text { Right } \\
\text { posterior }\end{array}$ & $\begin{array}{c}\text { Bilateral } \\
\text { posterior }\end{array}$ & $\begin{array}{c}\text { Total posterior } \\
\text { Percent of all 418 infarcts }\end{array}$ \\
\hline Basilar and multiple small emboli & 0 & 0 & 1 & 1 & $0.24 \%$ \\
Basilar artery & 0 & 0 & 20 & 20 & $4.78 \%$ \\
Other Cerebellar & 2 & 3 & 0 & 5 & $1.20 \%$ \\
PCA & 15 & 13 & 1 & 29 & $6.94 \%$ \\
PCA and multiple small emboli & 0 & 0 & 1 & 1 & $0.24 \%$ \\
PICA & 8 & 9 & 0 & 17 & $4.07 \%$ \\
PICA with multiple small emboli & 1 & 0 & 0 & 34 & $0.24 \%$ \\
Brainstem lacunar & 20 & 14 & 0 & 21 & $8.13 \%$ \\
Thalamic lacunar & 8 & 13 & 0 & $5.02 \%$
\end{tabular}

PCA, posterior cerebral artery; PICA, Posterior inferior cerebellar artery 
Table 3: Infarctions involving both anterior and posterior circulations. Simultaneous infarctions in MCA (middle cerebral artery) and PCA (posterior cerebral artery) territories, MCA and PICA (posterior inferior cerebellar artery) territories, etc. See text for further description of categories.

\begin{tabular}{lccccc}
\hline Category & $\begin{array}{c}\text { Left } \\
\text { combined } \\
\text { anterior and } \\
\text { posterior }\end{array}$ & $\begin{array}{c}\text { Right } \\
\text { combined } \\
\text { anterior and } \\
\text { posterior }\end{array}$ & $\begin{array}{c}\text { Bilateral } \\
\text { combined } \\
\text { anterior and } \\
\text { posterior }\end{array}$ & $\begin{array}{c}\text { Total } \\
\text { combined } \\
\text { anterior and } \\
\text { posterior }\end{array}$ & Percent of all 418 infarcts \\
\hline MCA and PCA & 1 & 2 & 0 & 3 & $0.72 \%$ \\
MCA and PICA & 0 & 0 & 1 & 1 & $0.24 \%$ \\
MCA/PCA watershed & 7 & 2 & 0 & 9 & $2.15 \%$ \\
MCA/PCA watershed and & 0 & 2 & 0 & 2 & $0.48 \%$ \\
multiple small emboli & 0 & 6 & 23 & 29 & $6.94 \%$ \\
Multiple small emboli & 0 & 6 & & & \\
\hline
\end{tabular}

MCA, middle cerebral artery; PCA, posterior cerebral artery; PICA, Posterior inferior cerebellar artery

the distribution of strokes within the brain, the value of quantitative data is illustrated by a study showing that 56 of 135 strokes in cardiac surgery patients $(41 \%)$ were in the posterior cerebral circulation, compared to 100 out of 503 strokes $(20 \%)$ in the control group..$^{10}$ The finding that strokes after cardiac surgery occurred twice as often in the posterior cerebral circulation suggests the possibility that a search for a cardiac source of thromboembolism is twice as worthwhile if a stroke is in the posterior cerebral circulation. Quantitative data strengthen this conclusion. That study was performed in a country with a national healthcare database. ${ }^{10}$ For countries without nationalized health care and such databases, it would be beneficial to have as large and as diverse as possible database of the distribution of strokes within the brain.

Our study can generate hypotheses with implications for stroke patient management. CTA to look for potentially retrievable clots is often performed, but this risks acute kidney injury, which may not be a worthwhile risk in patients with lacunar strokes. The finding that $30.8 \%$ of the strokes in our study were lacunar infarcts in the internal capsule, thalamus, or brainstem suggests that guidelines for when not to perform CTA might benefit from data of the neuroanatomical location of strokes.

Currently, there is insufficient data on the incidence of strokes in specific areas of the brain. If this data were available, linking it to longitudinal population-based incidence studies with full case-ascertainment and repeated outcome assessments could be used to determine prognosis for strokes in each location of the brain and to drive research into improving that prognosis. ${ }^{9}$ Quantitative data about the location of strokes within the brain could be useful in research on risk factors leading to strokes in specific areas of the brain and preventative strategies based on those risk factors. ${ }^{11}$ Quantitative data about the location of strokes within the brain from different parts of a country or different countries could be useful in research on the social determinants of health disparities. ${ }^{12}$
Our study was not designed to do the valuable research that could be done with large scale quantitative data about the location of strokes within the brain. Our study was a small pilot study of how the quantitative data could be gathered. We started with the database of a regional hospital stroke center and focused on the neuroradiological diagnosis, which we checked by review with a neurologist, who also identified the most useful categories. There could be other ways of categorizing strokes useful for different purposes. Our study was limited to acute ischemic strokes and excluded hemorrhagic strokes. Other studies could examine hemorrhagic as well as acute ischemic strokes. Our study allowed separating out embolic strokes on the basis of neuroradiological features, but did not allow separating out those embolic from the heart. Other studies could explore potentially important differences between cardioembolic strokes and other embolic strokes. There is need for large scale quantitative data-ie, "big data." Our small pilot study illustrates a methodology for collecting the data to substitute specific numerical quantitation for vague generalities about the neuroanatomical distribution of strokes. Such quantification can enable evidence-based data-driven improvements in the care of stroke patients.

\section{References}

1. Centers for Disease Control and Prevention: CDC. Stroke: Statistics and Facts. Available at: https://www. cdc.gov/stroke/facts.htm. Accessed June 20, 2020.

2. Yaghi S, Ishida K, Torres J, et al. SARS-CoV-2 and Stroke in a New York Healthcare System [published correction appears in Stroke. 2020 Aug;51(8):e179]. Stroke. 2020;51(7):2002-2011.

3. Pistoia F, Sacco S, Tiseo C, Degan D, Ornello R, Carolei A. The Epidemiology of Atrial Fibrillation and Stroke. Cardiol Clin. 2016;34(2):255-268. doi:10.1016/j. ccl.2015.12.002.

4. Sacco RL, Sur NB. Rising Incidence of Stroke in Pregnancy: A Call for Enhanced Screening and Prevention. J Am Coll Cardiol. 2020;75(2):191-193. doi:10.1016/j.jacc.2019.11.029 
5. Hurford R, Sekhar A, Hughes TAT, Muir KW. Diagnosis and management of acute ischaemic stroke. Pract Neurol. 2020;20(4):304-316.

6. Ropper AH, Samuels MA, Klein JP, Prasad S. Adams and Victor's Principles of Neurology. 11th ed. New York: McGraw-Hill; 2019.

7. Kumar V, Abbas AK, Aster JC. Robbins \& Cotran Pathologic Basis of Disease. 10th ed. Philadelphia: Elsevier; 2021.

8. Howard J, Singh A. Neurology Image-Based Clinical Review. New York: DemosMedical; 2017.

9. Ung D, Kim J, Thrift AG, et al. Promising Use of Big Data to Increase the Efficiency and Comprehensiveness of Stroke Outcomes Research. Stroke. 2019;50(5):13021309.

10. Pierik R, Uyttenboogaart M, Erasmus ME, Scheeren TWL, van den Bergh WM. Distribution of perioperative stroke in cardiac surgery. Eur J Neurol. 2019;26(1):184190.

11. Assarzadegan F, Tabesh H, Shoghli A, et al. Relation of Stroke Risk Factors with Specific Stroke Subtypes and Territories. Iran J Public Health. 2015;44(10):13871394.

12. Magwood GS, Ellis C, Nichols M, et al. Barriers and Facilitators of Stroke Recovery: Perspectives From African Americans With Stroke, Caregivers and Healthcare Professionals. J Stroke Cerebrovasc Dis. 2019;28(9):2506-2516.

\section{Author Affiliations}

Larry Nichols, $M D^{*}$; Joshua C. Bridgewater, $B S^{\prime}$;

Nicholas B. Wagner, $B S^{\prime}$; Mathew Karivelil, BS'; Himara

Koelmeyer, BS ; Denise Goings, $M S N^{*}$; and Thomas D.

Hope, $M D^{*}$

"Department of Pathology and Clinical Sciences Education, Mercer University School of Medicine, Macon, Georgia, USA

'Medical Student, Mercer University School of Medicine, Macon, Georgia, USA

${ }^{*}$ Stroke Program Coordinator, Medical Center Navicent Health, Macon, Georgia, USA 